\section{P09.02 REATTENDANCE RATES IN MEN PRESENTING WITH SYMPTOMS OF URETHRITIS - CAN POINT OF CARE TESTING FOR CHLAMYDIA AND GONORRHOEA IMPROVE OUTCOMES?}

${ }^{1,2} \mathrm{~N}$ Carter, ${ }^{1} \mathrm{M}$ Crofts, ${ }^{2} \mathrm{~F}$ Garrett, ${ }^{2} \mathrm{M}$ Clarke, ${ }^{2} \mathrm{~J}$ Nicholls, ${ }^{2} \mathrm{H}$ Wheeler, ${ }^{1} \mathrm{D}$ Sharp, ${ }^{1,2} \mathrm{P}$ Horner*. ' ${ }^{1}$ chool of Social and Community Medicine, University of Bristol, UK; ${ }^{2}$ Bristol Sexual Health Centre, University Hospitals Bristol NHS Trust, Bristol, UK

\subsection{6/sextrans-2015-052270.386}

Introduction Chlamydia and gonorrhoea are common causes of urethritis. Management is often based on an enhanced syndromic approach while awaiting results. This can necessitate prescribing to cover a range of potential pathogens, and uncertainty for patients. Point of care testing (POCT) for chlamydia and gonorrhoea in men with symptoms of urethritis could alter care pathways and reduce reattendance in these patients. The aim of this study was to measure reattendance rates in men presenting with symptoms of urethritis and develop a decision tree care pathway model in order to estimate potential benefits of replacing standard nucleic acid amplification testing with POCT.

Methods All men with urethritis symptoms presenting over a three month period were identified using electronic patient records. Urethritis was defined as $\geq 5 \mathrm{pmnls} / \mathrm{hpf}$ on a Gram stained urethral smear. Reattendances within 30 days of initial clinic visit and reasons for reattendance were recorded for both microscopy-positive and negative groups. Review of literature was used to provide estimates of improved outcomes if the chlamydia/gonorrhoea POCT result was available prior to the clinical consultation.

Results 431 men with urethritis symptoms were identified in a 3 month period. 192 had confirmed urethritis on initial microscopy. $31 \%$ of microscopy-positive men and $42 \%$ of microscopynegative men reattended at least once within 30 days of initial visit. Common reasons for reattendance were early morning smear (20\%), persistent symptoms (18\%), results (16\%) and gonorrhoea test of cure $(9 \%)$. It was estimated that POCT could reduce microscopy by $25 \%$ and repeat reattendance following treatment by $75 \%$ through improved pathogen-directed treatment and the introduction of gonorrhoea POCT sample drop-off as a test of cure.

Conclusion This service evaluation using decision tree care pathway modelling has identified high reattendance rates in men with urethritis symptoms which POCT has the potential to reduce substantially.

Disclosure of interest statement $\mathrm{PH}$ has received funding from Cepheid directly and indirectly for lecturing on point of care testing and undertaking research on the cost effectiveness of their CT/NG assay. Has also received payment from Atlas Genetics for an article in the Parliamentary Review on the benefits of point of care technology in improving the cost effectiveness of sexual health services. Has also received an honorarium from Hologic for an education talk on STI diagnostics.

NC, MC, FG, MC, JN, HW, no conflicts of interest declared.

\section{P09.03 TREATMENT OF MYCOPLASMA GENITALIUM WITH AZITHROMYCIN 1 G IS LESS EFFICACIOUS AND ASSOCIATED WITH INDUCTION OF MACROLIDE RESISTANCE COMPARED TO A 5-DAY REGIMEN}

${ }^{1,2} \mathrm{P}$ Horner*, ${ }^{1} \mathrm{~S}$ Ingle, ${ }^{1} \mathrm{~F}$ Garrett, ${ }^{2} \mathrm{~K}$ Blee, ${ }^{3} \mathrm{FYS}$ Kong, ${ }^{4} \mathrm{H}$ Moi. ${ }^{1}$ School of Social and Community Medicine, University of Bristol, UK; ${ }^{2}$ Bristol Sexual Health Centre, University Hospitals Bristol NHS Trust, Bristol, UK; ${ }^{3}$ Centre for Epidemiology and Biostatistics, Melbourne School of Population and Global Health, University of Melbourne, Australia; ${ }^{4}$ Olafia Clinic, Oslo University Hospital, Institute of Medicine, University of Oslo, Norway

\subsection{6/sextrans-2015-052270.387}

Introduction Mycoplasma genitalium (MG) is an emerging important STI. Failure rates with azithromycin $1 \mathrm{~g}$ appear to be increasing. This may be due to the emergence of macrolide antimicrobial resistance as a consequence of extensive use of azithromycin $1 \mathrm{~g}$. An extended regimen of azithromycin $500 \mathrm{mgs}$ on day one then $250 \mathrm{mgs}$ daily for 4 days (5 day regimen) was introduced in the 1990s for treatment of MG and has high efficacy rates (if no pre-existing macrolide resistance) and is less associated with induction of macrolide resistance. There are no comparative trials of the two regimens. We undertook a metaanalysis of MG treatment studies using the two azithromycin regimens to determine which is more effective.

Methods Medline was used to identify published articles including the search terms Mycoplasma genitalium and resistance up to March 2015. Treatment studies using azithromycin $1 \mathrm{~g}$ or 5 days were identified in which patients were initially assessed for macrolide resistance genetic mutations, and those who failed were again resistance genotyped were selected. Sensitivity analyses included only patients without prior treatment.

Results Six studies were identified totaling 424 patients of whom $78(18.4 \%)$ had received the 5 dy regimen. Only one person failed the 5 day regimen and no resistance was detected. Compared to the 5 day regimen, azithromycin $1 \mathrm{~g}$ had a higher risk of failure (difference: $12.9 \%, 95 \% \mathrm{CI}: 8.4 \%, 17.3 \%$ ) and more developed macrolide resistance (risk difference: 12.1\% $(8.7 \%, 15.6 \%)$. The 5 day regimen included 52 patients with prior doxycycline treatment when these were excluded sensitivity analysis showed a failure risk difference of $10.3 \%(2.1 \%$, 18.6\%). Resistance risk did not change.

Conclusion Azithromycin $1 \mathrm{~g}$ is more likely to result in treatment failure and the development of macrolide antimicrobial resistance than $500 \mathrm{mgs}$ on day one then $250 \mathrm{mgs}$ daily for 4 days.

Disclosure of interest statement $\mathrm{PH}$ : - Has received funding for providing expert advice on M. genitalium diagnostics. HM - has received a honorarium and travel expenses from Becton Dickinson for a lecture on Mycoplasma genitalium. SI, FG, FK and KB - none to declare.

\section{P09.04 TRICHOMONAS VAGINALIS AND MYCOPLASMA GENITALIUM: AGE-SPECIFIC PREVALENCE AND DISEASE BURDEN IN MEN ATTENDING A SEXUALLY TRANSMITTED INFECTIONS CLINIC IN AMSTERDAM, THE NETHERLANDS}

${ }^{1} \mathrm{C}$ van der Veer*, ${ }^{2} \mathrm{MS}$ van Rooijen, ${ }^{2,3,4} \mathrm{HJC}$ de Vries, ${ }^{1} \mathrm{M}$ Himschoot, ${ }^{1} \mathrm{SM}$ Bruisten. ${ }^{1}$ Public Health Laboratory, GGD Amsterdam, The Netherlands; ${ }^{2}$ STI Clinic, GGD Amsterdam, The Netherlands; ${ }^{3}$ Department of Dermatology, Academic Medical Centre, University of Amsterdam, The Netherlands; ${ }^{4}$ Centre for Infection and Immunity Amsterdam (CINIMA), Academic Medical Centre, University of Amsterdam, The Netherlands

10.1136/sextrans-2015-052270.388 